\title{
Identifying and treating group A streptococcal pharyngitis in children
}

\author{
Michelle Science MD MSc, Ari Bitnun MD MSc, Warren Mclsaac MD MSc
}

See related research article on page 23 and at www.cmaj.ca/lookup/doi/10.1503/cmaj.140772; see also case on page 50 and at www.cmaj.ca /lookup/doi/10.1503/cmaj.140155

$\mathrm{P}$ haryngitis is common and a frequent presentation to family physicians and pediatricians. ${ }^{1}$ Most of these infections are caused by viruses, but about $20 \%-40 \%$ of cases in children are due to group A streptococcal infection. ${ }^{2,3}$ Differentiating between streptococcal and viral causes has proven difficult, because individual signs and symptoms are not sufficiently discerning. ${ }^{4}$ Thus, some expert guidelines recommend microbiologic testing. ${ }^{5}$ However, given the rarity of complications such as rheumatic fever in high-resource countries, the delay in obtaining throat culture results, an the large number of negative tests, physicians have frequently used a selective strategy of testing in some cases and starting antibiotic therapy before test results are received in others. ${ }^{1}$

Clinical prediction rules have been developed to help clinicians predict the likelihood of streptococcal infection more accurately, and to guide selective treatment decisions. Although several such rules have been developed, ${ }^{6}$ is has been suggested that none has the necessary sensitivity or specificity to eliminate the need for children to undergo microbiologic testing. Cohen and colleagues ${ }^{7}$ have reviewed the available clinical prediction rules for diagnosing and managing streptococcal pharyngitis in children and directly compared their diagnostic accuracy. They found that each clinical prediction rule required some degree of adaptation to incorporate rapid antigen detection testing. They then applied the adapted rules to patients in a validation cohort to classify each patient as being at low (no further testing or antibiotic therapy), intermediate (rapid antigen detection testing for all, antibiotics for those with positive test results) and high risk (empiric treatment) of streptococcal infection. With this strategy, they were unable to identify patients at low or high risk in whom microbiologic testing could be avoided.

Microbiologic testing for group A streptococcus includes culture-based approaches and rapid antigen detection tests. Throat culture is considered the gold standard and has a high sensitivity, but it may be associated with higher cost and delayed treatment. Rapid antigen detection tests are highly specific (95\%) and can aid in making immediate clinical decisions, but they are limited by low sensitivity $(70 \%-90 \%),{ }^{8}$ which varies with disease spectrum, size of the bacterial inoculum and the person performing the test. A limitation of both test modalities is the inability to distinguish between colonization or carriage (occurring in $5 \%-20 \%$ of children age 3-15 yr) and infection.

Cohen and colleagues' study is the first to use a validation cohort to evaluate the utility of various clinical prediction rules specifically in children. However, there are two caveats to keep in mind when interpreting the results. First, the children in the validation cohort were recruited by a relatively select group of academic French pediatricians in urban areas. The severity of illness in the validation cohort may have been toward the sicker end of the spectrum. The prevalence of positive test results in the low- and high-risk strata were similar using the various clinical prediction rules. Second, the study did not attempt to discern age-related differences in the utility of the clinical prediction rules. Achieving results for older children similar to those seen in adults is plausible. ${ }^{9}$ Despite these limitations, however, this paper highlights the need for further guidance in diagnosing and treating pharyngitis in children.

Owing to a substantial decline in the incidence of suppurative and nonsuppurative complications of group A streptococcal infection in high-resource settings, the importance of univer-

\section{- KEY POINTS}

- Current clinical prediction rules are insufficiently sensitive and specific to guide management of acute pharyngitis in children.

- Increasing antibiotic resistance and the rarity of complications associated with group A streptococcal pharyngitis in high-resource countries suggest that a new approach is needed in the approach to acute pharyngitis.

- The time has come for a Canadian strategy that will take into account safety, cost, antimicrobial resistance and physician practices. 
sally treating pharyngitis has been questioned. ${ }^{10}$ However, a Cochrane review has shown that antibiotic treatment of sore throat reduces the risk of otitis media and peritonsillar abscess, the duration of symptoms and the rate of acute rheumatic fever. ${ }^{11,12}$ In the United Kingdom, a reduction in prescribing antibiotics may have been a contributing factor for increased admissions for peritonsillar and retropharyngeal abscesses. ${ }^{13}$ Currently, many European guidelines recommend antibiotic treatment only for high-risk patients, whereas North American guidelines focus on treatment for all patients with group A streptococcal pharyngitis. ${ }^{10}$

So, which patients with sore throat should receive treatment? Given that most sore throats are caused by viruses, giving treatment to all patients would result in unnecessary antibiotic use in an era of increasing antimicrobial resistance. ${ }^{3}$ Having all patients undergo testing will reduce antibiotic exposure, but may be less desirable to physicians who have to follow-up with patients after their appointments to provide results or call in prescriptions. This strategy also involves higher cost and may lead to increased symptom duration.

Cohen and colleagues argue against a selective strategy for testing and treating owing to insufficient diagnostic accuracy of the clinical prediction rules. However, they make no comment on the cost-effectiveness of a universal testing strategy. Furthermore, for any strategy to be effective, it must be simple and have buy-in from frontline physicians. Data from the United States suggest that only half of physicians perform throat swabs, and half write a prescription before the results of the test are available. ${ }^{1}$ Similarly, a recent paper from Israel showed that only $54 \%$ of physicians adhered to guidelines recommending testing in all children. ${ }^{14}$ Cohen and colleagues do not address this question.

Currently, no Canadian consensus guideline on the approach to sore throat in children exists. We need to define a strategy that is safe, cost-effective and useful in both urban and rural settings. Canadian health organizations for family physicians, pediatricians and other relevant stakeholders should work together to define such a strategy. Whatever strategy they recommend should be accompanied by ongoing surveillance for group A streptococcal pharyngitis and its complications, antibiotic use and resistance rates. Further research is needed to determine whether clinical prediction rules could be useful, perhaps by focusing on specific age strata.

\section{References}

1. Linder JA, Bates DW, Lee GM, et al. Antibiotic treatment of children with sore throat. JAMA 2005;294:2315-22.

2. Shaikh N, Leonard E, Martin JM. Prevalence of streptococcal pharyngitis and streptococcal carriage in children: a meta-analysis. Pediatrics 2010;126:e557-64.

3. Kronman MP, Zhou C, Mangione-Smith R. Bacterial prevalence and antimicrobial prescribing trends for acute respiratory tract infections. Pediatrics 2014;134:e956-65.

4. Shaikh N, Swaminathan N, Hooper EG. Accuracy and precision of the signs and symptoms of streptococcal pharyngitis in children: a systematic review. J pediatr 2012;160:487-493.e3.

5. Shulman ST, Bisno AL, Clegg HW, et al. Clinical practice guideline for the diagnosis and management of group A streptococcal pharyngitis: 2012 update by the Infectious Diseases Society of America. Clin Infect Dis 2012;55:1279-1282.

6. Maguire JL, Kulik DM, Laupacis A, et al. Clinical prediction rules for children: a systematic review. Pediatrics 2011;128: e666-77.

7. Cohen JF, Cohen R, Levy C, et al. Selective testing strategies for diagnosing group A streptococcal infection in children with pharyngitis: a systematic review and prospective multicentre external validation study. CMAJ 2014 Dec. 8 [Epub ahead of print].

8. Stewart EH, Davis B, Clemens-Taylor BL et al. Rapid antigen group A streptococcus test to diagnose pharyngitis: a systematic review and meta-analysis. PLoS ONE 2014;9:e111727.

9. Fine AM, Nizet V, Mandl KD. Large-scale validation of the Centor and McIsaac scores to predict group A streptococcal pharyngitis. Arch Intern Med 2012;172:847-52.

10. Van Brusselen D, Vlieghe E, Schelstraete P, et al. Streptococcal pharyngitis in children: to treat or not to treat? Eur J Pediatr 2014; 173:1275-83.

11. Spinks A, Glasziou PP, Del Mar CB. Antibiotics for sore throat. Cochrane Database Syst Rev 2013;11:CD000023.

12. Madden S, Kelly L. Update on acute rheumatic fever: It still exists in remote communities. Can Fam Physician 2009;55:475-8.

13. Lau AS, Upile NS, Wilkie MD, et al. The rising rate of admissions for tonsillitis and neck space abscesses in England, 1991-2011. Ann R Coll Surg Engl 2014;96:307-10.

14. Urkin J, Allenbogen M, Friger M, et al. Acute pharyngitis: low adherence to guidelines highlights need for greater flexibility in managing paediatric cases. Acta Paediatr 2013;102:1075-80.

Affiliations: Division of Infectious Diseases, Department of Paediatrics (Science, Bitnun), The Hospital for Sick Children; Granovsky-Gluskin Family Medicine Centre, Ray D. Wolfe Department of Family Medicine (McIsaac), Mount Sinai Hospital, University of Toronto; Department of Community and Family Medicine (McIsaac), University of Toronto, Toronto, Ont.

Contributors: Each of the authors conceived the idea for the manuscript. Michelle Science drafted the manuscript, which was revised by Ari Bitnun and Warren McIsaac for important intellectual content. All of the authors approved the final version submitted for publication. 